\title{
Ischemic expansion during acute myocardial infarction and reversal by coronary reperfusion
}

\begin{abstract}
Previous studies have shown that infarct expansion occurs at least 1 day after a large transmural infarction. To assess whether regional left ventricular expansion is evident within hours of an acute myocardial infarction, 25 adult mongrel dogs underwent left circumflex coronary artery occlusion for 2 hours and 22 of these were subsequently reperfused. Two-dimensional echocardiography was used to record left ventricular topography and function at baseline, at 2 hours of occlusion, and following reperfusion. Short-axis midpapillary echocardiograms were analyzed using a microcomputer digitizing routine by establishing a 360 -degree circumferential map of the left ventricle. The central ischemic zone was defined as that region with the most depressed contractility after 2 hours of occlusion, and the normal zone was set at 180 degree away from the central ischemic zone. Endocardial and epicardial segment lengths and wall thickness were measured for both the normal zone and the central ischemic zone at end diastole. After 2 hours of occlusion, diastolic central ischemic endocardial $(1.3 \pm 0.05$ to $1.42 \pm 0.04 \mathrm{~cm}, p<0.01)$ and central ischemic epicardial $(1.84 \pm 0.06$ to $1.93 \pm 0.06 \mathrm{~cm}$, $p<0.05$ ) segment lengths were significantly increased. There were no significant changes in segment lengths or wall thickness in the normal zone. After 2 hours of occlusion, there was significant diastolic left ventricular (LV) dilatation ( $L V$ area increased from $18.2 \pm 1.3$ to $21.0 \pm 1.3 \mathrm{~cm}^{2}, p<0.01$ ). Furthermore, central ischemic endocardial and epicardial segment length changes from baseline to occlusion correlated significantly with LV dilatation $(f=0.56$, $p<0.003 ; r=0.55, p<0.004$ respectively). Following 2 hours of reperfusion, central ischemic endocardial, epicardial, and LV area decreased significantly and approached baseline values. We conclude that ischemic expansion exists and can be identified using two-dimensional echocardiography; that it occurs early following coronary artery occlusion; and that it is reversed by reperfusion. (AM HEART J 1992;123:1456.)
\end{abstract}

Michael J. Lim, BS, Beth L. Karolle, BS, John C. Wood, BS, and

Andrew J. Buda, MD. New Orleans, La., and Ann Arbor, Mich.

Infarct expansion is a relatively common complication following an acute myocardial infarction and has been reported to occur in $70 \%$ of all transmural infarcts. ${ }^{1}$ However, early reperfusion produced by thrombolytic therapy may salvage myocardium and

F'rom the Cardiology Section, Department of Medicine, Tulane University School of Medicine, New Orleans; and the Division of Cardiology, Department of Internal Medicine, University of Michigan Medical School, Ann Arbor.

Supported by Grant R01 HL 34691 from the National Heart, Lung, and Blood Institute, National Institutes of Health, Bethesda, Md. Michael Lim was the recipient of a Student Research Grant from the University of Michigan Medical School, Ann Arbor. Beth Karolle was the recipient of a Student Research Fellowship from The American Heart Association of Michigan, Lathrup Villiage, Michigan.

Received for publication Oct. 4, 1991; accepted Nov. 25, 1991.

Reprint requests: Andrew J. Buda, MD, Section of Cardiology, Tulane University School of Medicine, 1430 Tulane Ave., New Orleans, LA 70112. 4/1/36276 limit subsequent expansion. Moreover, experimental studies ${ }^{2,3}$ have demonstrated that coronary reperfusion prevents infarct expansion, regardless of its ability to successfully salvage myocardium. Thus the topographic changes that occur immediately following the onset of ischemia may be of greater significance in the early evolution of an acute myocardial infarction.

For many years, it has been known that coronary occlusion leads to distinctive changes in myocardial contraction patterns. ${ }^{4}$ Several investigators ${ }^{5,6}$ have reported alterations in regional ventricular geometry that immediately follow the onset of myocardial ischemia, including the familiar systolic bulging. Studies using sonomicrometry ${ }^{7,8}$ have also shown that end-diastolic segment lengths increase following coronary artery occlusion. Accordingly, we hypothesized that these end-diastolic changes are a form of expansion that we will term "ischemic expansion." 


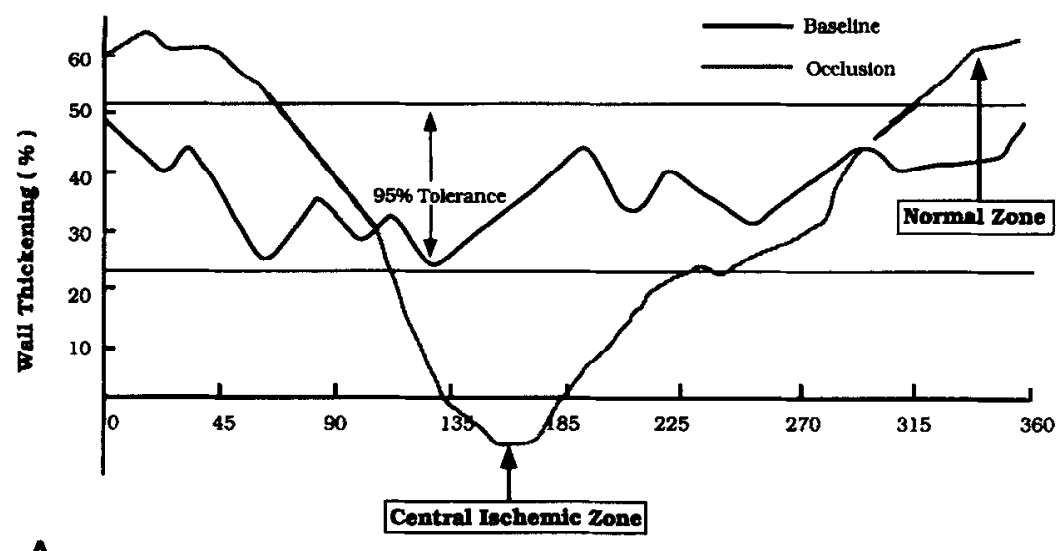

A

Circumferential Angle $\left({ }^{\circ}\right)$

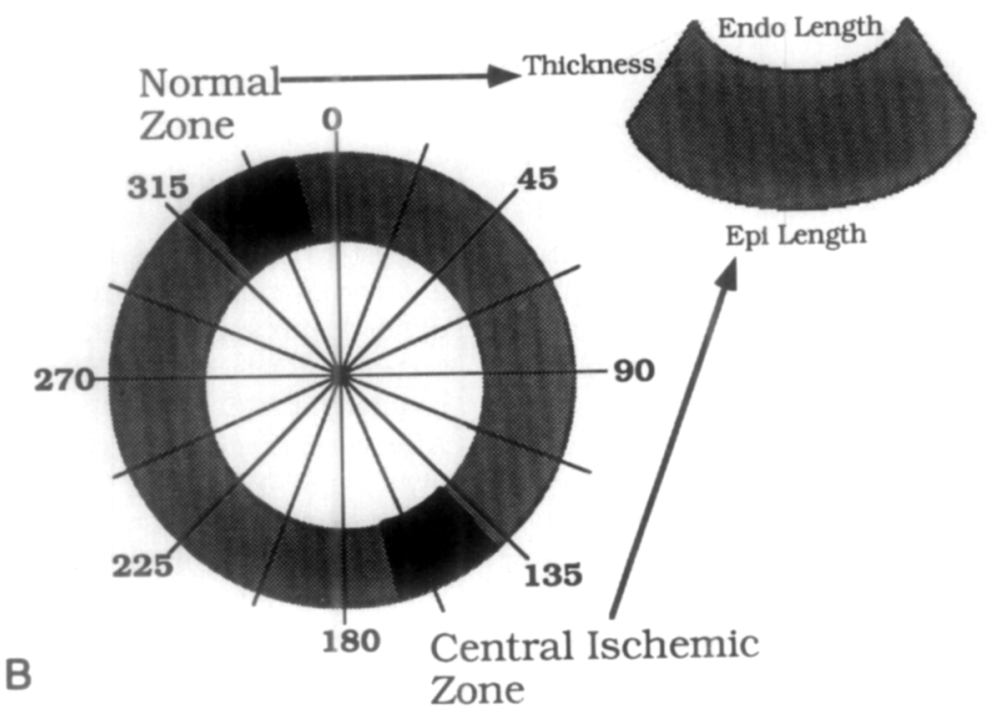

Fig. 1. Illustrated is the methodology used in analyzing the echocardiographic images. A, Schematic representation of a wall thickening map generated from the short-axis left ventricular echocardiogram. The baseline wall thickening is plotted in the solid line and $95 \%$ tolerance limits are drawn for this curve. The occlusion wall thickening is then plotted on the same axis and the central ischemic zone and the normal zone are subsequently defined. B, Schematic of the short-axis image with the central ischemic and normal zones highlighted. Endocardial length, epicardial length, and wall thickness are measured for each of these zones as depicted.

The end-diastolic regional and global left ventricular changes that constitute ischemic expansion have yet to be well characterized or understood. Therefore the aim of this study was twofold: first, to use a quantitative two-dimensional echocardiographic method to measure regional segmental and global changes in the ischemic ventricle and second, using this approach, to examine ischemic expansion following subsequent reperfusion.

\section{METHODS}

Guidelines of The University of Michigan Committee on the Use and Care of Animals were followed for all parts of the experimental procedure. The University of Michigan is accredited by the American Association of Accreditation of Laboratory Animal Care.

Animal preparation. Adult male mongrel dogs were initially anesthetized with thiamylal $(20 \mathrm{mg} / \mathrm{kg})$ intravenously, followed by pentoharbital sodium $(30 \mathrm{mg} / \mathrm{kg})$ as needed, and ventilated with a Harvard respirator (Harvard Apparatus Inc., S. Natick, Mass.). Catheters were placed in the left internal jugular vein for fluid and drug administration, in the left carotid artery for mean arterial pressure measurements, and in the right femoral artery for the withdrawal of reference arterial blood samples. A left thoracotomomy via the fifth intercostal space was used to access the heart, which was then suspended within a pericar- 
Table I. Myocardial segment length changes following occlusion

\begin{tabular}{lccccccc}
\hline & \multicolumn{3}{c}{ Normal zone } & & & \multicolumn{2}{c}{ Central ischemic zone } \\
\cline { 2 - 3 } & Baseline & Occlusion & $\mathrm{p}$ Value & & Baseline & Occlusion & $\mathrm{p}$ Value \\
\hline Endocardium $(\mathrm{cm})$ & $1.20 \pm 0.04$ & $1.26 \pm 0.05$ & NS & & $1.33 \pm 0.05$ & $1.42 \pm 0.04$ & $<0.01$ \\
Epicardium $(\mathrm{cm})$ & $1.70 \pm 0.04$ & $1.77 \pm 0.05$ & NS & & $1.84 \pm 0.06$ & $1.93 \pm 0.06$ & $<0.05$ \\
\hline
\end{tabular}

$N S$, Not statistically significant.

Table II. Myocardial segment length changes following reperfusion

\begin{tabular}{|c|c|c|c|c|c|c|}
\hline & \multicolumn{3}{|c|}{ Normal zone } & \multicolumn{3}{|c|}{ Central ischemic zone } \\
\hline & Occlusion & Keperfusion & $\mathrm{p}$ Value & Occlusion & Reperfusion & p Value \\
\hline Endocardium $(\mathrm{cm})$ & $1.26 \pm 0.05$ & $1.26 \pm 0.05$ & NS & $1.42 \pm 0.04$ & $1.30 \pm 0.04$ & $<0.01$ \\
\hline Epicardium $(\mathrm{cm})$ & $1.77 \pm 0.05$ & $1.77 \pm 0.05$ & NS & $1.93 \pm 0.06$ & $1.84 \pm 0.05$ & $<0.05$ \\
\hline
\end{tabular}

Abbreviation as in Table I.

dial cradle. A catheter was placed in the left atrial appendage for pressure monitoring and microsphere injection. The left circumflex artery was then isolated proximal to the first marginal branch and a snare occluder was positioned on the artery with an ultrasonic flow probe placed proximal to the occluder.

Ischemia-reperfusion protocol. A number of study protocols were used to evaluate the significance of ischemia and varying durations of reperfusion on expansion. A subset of experiments consisted of only a 2 -hour occlusion period $(n=3)$. Other experiments involved a 2-hour occlusion period followed by a $2-(n=7), 3-(n=2)$, or 4 -hour reperfusion period $(n=13)$. Short axis two-dimensional echocardiograms were obtained of the left ventricle at the midpapillary muscle level and hemodynamics were also recorded at baseline, at the end of occlusion, and at the end of reperfusion. Following completion of the protocol, a 60 $\mathrm{cm}^{3}$ bolus of Evan's blue dye was infused into the left atrium while the left circumflex artery was again occluded to assess the myocardium at risk. The animal was put to death with an intravenous bolus of saturated $\mathrm{KCl}$ solution and the heart was excised. The left ventricle was isolated and was subsequently sliced into six to nine transverse sections. Triphenyltetrazolium (TTC) staining was used to assess the necrotic area. Risk area and infarct size were determined by planimetry of the individual slices of the left ventricle.

Standard microsphere techniques were used to determine regional myocardial blood flow at baseline, at the end of occlusion, and at the end of reperfusion. Briefly, approximately $1-2 \times 10^{6}$ radioactive microspheres $(15 \mu \mathrm{m}$ diameter) were injected into the left atrium while simultaneous reference arterial samples were withdrawn from the femoral artery for subsequent measurement of myocardial blood flow using the reference withdrawal method. After excision of the heart, six to nine transverse slices, approximately $5 \mathrm{~mm}$ thick, were made to divide up the left ventricle. Two adjacent transverse slices at the midpapillary muscle level were then selected and a circumferential coordinate system was assigned that divided each slice into 16 sectors (22.5 degrees per sector). The central ischemic and normal zones were then defined and each segment was divided into endocardial, midmyocardial, and epicardial sections. Each sample was weighed and subsequently counted for radioactivity on a gamma scintillation counter. After correcting for background and overlap error, absolute myocardial blood flow was calculated by the equation: $\mathrm{Qm}=(\mathrm{Cm} \times \mathrm{Qr} / \mathrm{Cr})$, where $\mathrm{Qm}=$ myocardial blood flow (in milliliters per minute), $\mathrm{Cm}=$ counts per minute in the tissue sample, $\mathrm{Qr}=$ withdrawal rates of the reference sample (in milliliters per minute), and $\mathrm{Cr}=$ counts per minute in the reference arterial sample.

Echocardiographic analysis. Regional myocardial wall thickening was assessed using a Microsonics IBM-AT based video digitizing system (IBM Corp., Atlanta, Ga.) using the techniques previously reported from our laboratory ${ }^{9},{ }^{10}$ Briefly, a 360 -degree circumferential map was assigned to the left ventricle using a fixed diastolic center of mass and defining the posterior papillary muscle as 135 degrees. The endocardial and epicardial borders were then traced for three consecutive end-systolic and end-diastolic frames. From this analysis, wall thickening, extent of dysfunction, degree of dysfunction, diastolic left ventricular (LV) area, systolic LV area, and the area ejection fraction were calculated.

The method used to evaluate ischemic expansion is depicted in Fig. 1. The global wall thickening map was used to define the areas of least and greatest reduction in the percent wall thickening from the baseline time point to the end of occlusion. A 33.75-degree slice of myocardium containing the greatest dysfunctional region of the left ventricle was defined as the central ischemic zone. The normal zone was subsequently defined as a 33.75 -degree slice of myocardium that was exactly 180 degrees removed from the central ischemic zone. After defining the zones, the echo images and their circumferential maps were recalled and 


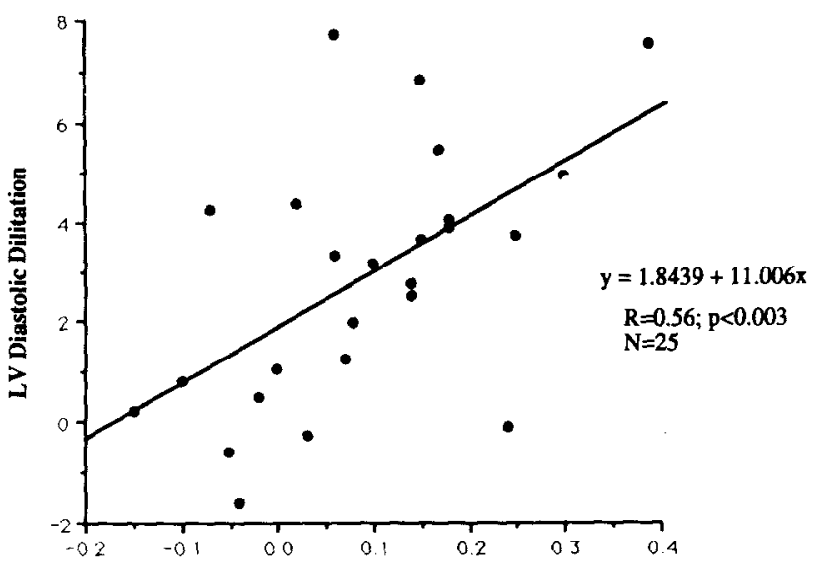

A

Endocardial Segment Length Change (cm)

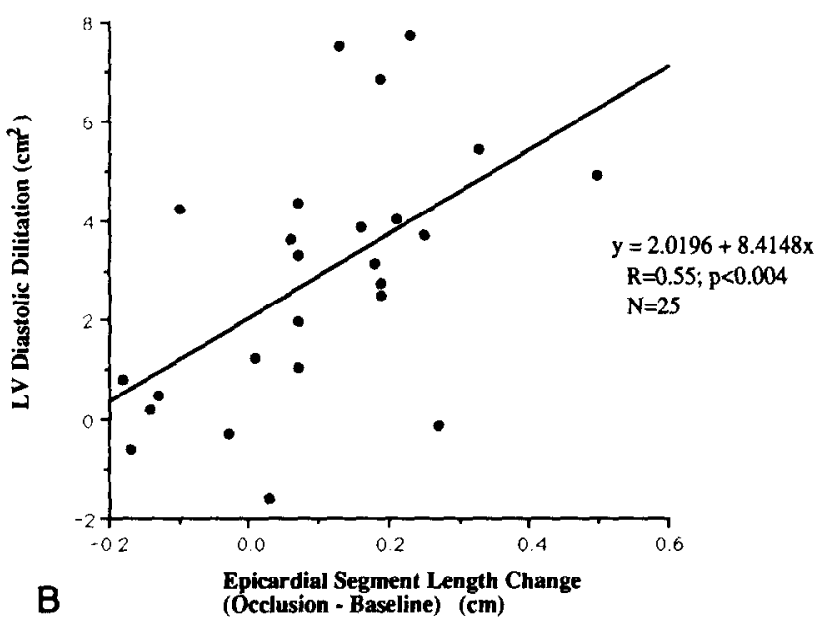

Fig. 2. Correlation between changes in endocardial (A) and epicardial (B) length and left ventricular dilatation from baseline to occlusion.

the endocardial and epicardial segment lengths and zone thicknesses were measured. Each measurement was averaged over three consecutive end-diastolic frames.

Statistical analysis. All data are expressed as mean \pm SEM. An analysis of variance (ANOVA) was used to compare the differences between the different ischemia-reperfusion protocols. Paired $t$ tests with a Bonferroni correction were used to assess the differences between time points. Standard linear regression analysis was used to determine the correlations between variables. The probability $(p)$ was considered to be statistically significant when less than 0.05 .

\section{RESULTS}

Differences between ischemia-reperfusion protocols. When the different ischemia-reperfusion studies were compared, no significant differences in hemodynamics, regional blood flow, or in measures of ischemic expansion for the baseline, end-occlusion, and the

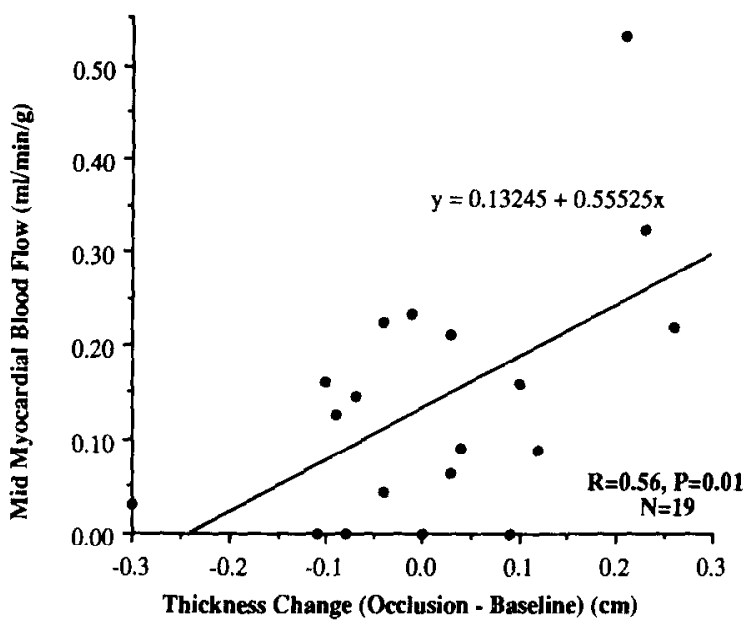

Fig. 3. Correlation of midmyocardial blood flow with the change in thickness in the central ischemic segment (baseline to occlusion).

end-reperfusion time points were observed. Therefore all of the protocols were pooled for statistical analysis.

Effect of coronary occlusion. Following 2 hours of left circumflex occlusion, heart rate increased from $136 \pm 3$ to $142 \pm 4$ beats $/ \mathrm{min}(p=\mathrm{NS})$. Mean arterial pressure significantly decreased from $129 \pm 4$ to $119 \pm 4 \mathrm{~mm} \mathrm{Hg}(p<0.05)$, and mean left atrial pressure increased from $4 \pm 1$ to $6 \pm 1 \mathrm{~mm} \mathrm{Hg}$ $(p<0.01)$. Table I summarizes the changes in segment length following occlusion. Both the endocardial and epicardial segment lengths in the central ischemic zone were significantly increased from their baseline values. Conversely, the segment lengths in the normal zone were not significantly changed from their baseline values. There was significant left ventricular dilatation following 2 hours of occlusion, with the left ventricular area increasing from $18.1 \pm 1.3$ $\mathrm{cm}^{2}$ to $21.0 \pm 1.3 \mathrm{~cm}^{2}(p<0.01)$. When the change in central ischemic segment length was compared with the change in left ventricular area, a significant linear correlation was found for both the endocardial segment length change and the epicardial segment length change (Fig. 2). There was no correlation found between changes in the normal zone segment length and the change in left ventricular area.

The thickness of the central ischemic zone or the normal zone was not significantly changed after the occlusion period (ischemic zone: $0.89 \pm 0.03$ to $0.91 \pm 0.03 \mathrm{~cm}, p=\mathrm{NS}$; normal zone: $0.90 \pm 0.03$ to $0.93 \pm 0.04 \mathrm{~cm}, p=\mathrm{NS}$ ). However, when the change in the central ischemic zone thickness was compared with the central ischemic blood flow, a significant linear correlation was found between the change in 


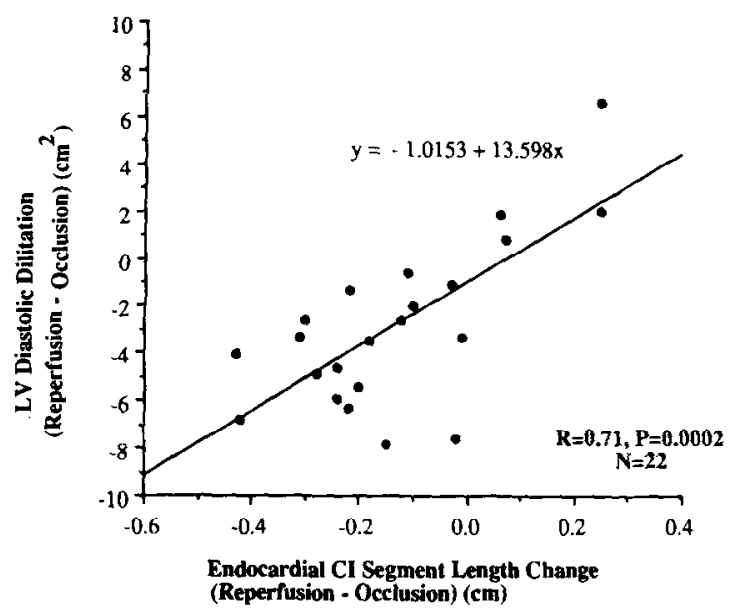

Fig. 4. Correlation between the change in endocardial central ischemic segment length (reperfusion to occlusion) and the change in left ventricular diastolic dilatation (occlusion to reperfusion).

central ischemic segment thickness and the subendocardial $(r=0.50, p=0.03)$, subepicardial $(r=0.53$, $p=0.02)$, and midmyocardial blood flow $(r=0.56$, $p=0.01$ ) (Fig. 3). There was no correlation between the normal zone thickness changes and regional blood flow $(r=0.1, p=\mathrm{NS})$.

Effect of reperfusion. Following reperfusion, heart rate slightly increased from $142 \pm 4$ to $147 \pm 6$ beats/min $(p=\mathrm{NS})$. Mean arterial pressure was significantly lower than during occlusion $(119 \pm 4$ versus $100 \pm 4 \mathrm{~mm} \mathrm{Hg} ; p<0.001$ versus occlusion, $p<0.0001$ versus baseline), and mean left atrial pressure increased from $6 \pm 1$ to $8 \pm 1 \mathrm{~mm} \mathrm{Hg}$ ( $p=$ NS versus occlusion; $p<0.001$ versus baseline). Table II summarizes the change in the segment lengths from the occlusion to the reperfusion time points. The endocardial and epicardial central ischemic segment lengths decreased significantly from occlusion, nearly returning to baseline values. Reperfusion did not alter the segment lengths in the normal zone.

The LV dilatation that was present at the end of the occlusion period was significantly reduced, with the $\mathrm{LV}$ area decreasing from $21 \pm 1.3 \mathrm{~cm}^{2}$ to 17.8 $\pm 1.3 \mathrm{~cm}^{2}(p<0.01)$. A significant linear correlation was again found between the decrease in the central ischemic endocardial segment length and the reduction of $\mathrm{LV}$ dilatation (Fig. 4). Thus the reduction in the central ischemic segment length contributed to the reduction in ventricular dilatation. However, no correlation was observed between the decrease in the epicardial segment length and the reduction of $\mathrm{LV}$ dilatation.

When the change in thickness of the central ischemic segment was compared with the regional myocardial blood flow, a significant linear correlation was found for the subendocardial $(r=0.69, p=$ $0.003)$, midmyocardial $(r=0.52, p=0.03)$, and subepicardial central ischemic blood flow $(r=0.51$, $p=0.05$ ) (Fig. 5). This suggests that the extent of reperfused blood flow determined the thickness of the postischemic myocardium.

Relationship to infarct size. A broad range of infarct sizes and risk areas was observed in this study. The percent infarct/left ventricle varied from $2.9 \%$ to $39.1 \%$ (mean $=17.8 \%, \mathrm{SEM}=1.95)$, and the percent infarct/risk area varied from $5.5^{\circ}, \mathrm{c}$ to $79.7^{\circ} \mathrm{c}$ (mean $=44.5 \%, \mathrm{SEM}=4.5$ ), while the risk areas ranged from $9.6 \%$ to $79.7 \%$ (mean $=44.5 \%, \mathrm{SEM}=$ 4.5). The changes in endocardial and epicardial segment lengths did not correlate with percent infarct/ left ventricle, percent infarct/risk area, or risk area.

\section{DISCUSSION}

It is well established that infarct expansion occurs within days of a large transmural myocardial infarct. However, the topographic changes that occur in the left ventricle during the early stages of infarction and following reperfusion are not well characterized. Accordingly, we hypothesized that regional LV expansion can also occur within hours of coronary occlusion. The important findings of the present study were that myocardial segment length in both the endocardial and epicardial layers of the ischemic region of the left ventricle increased during the episode of severe ischemia. This regional myocardial lengthening in the ischemic zone in turn contributed to the significant $\mathrm{LV}$ dilatation that was present at the end of the ischemic period. On the other hand, coronary reperfusion reversed this regional dilatation, with the reperfused segment length returning to its baseline value accompanied by a significant reduction in ventricular dilatation. These findings support the hypothesis that regional ventricular expansion occurs during prolonged severe ischemia and is reversed following reperfusion. In an effort to distinguish this pathophysiologic phenomenon from infarct expansion, we have termed this functional change ischemic expansion.

Infarct expansion. It is important to distinguish ischemic expansion from the well characterized phenomenon of infarct expansion. Infarct expansion has been shown to occur in $35 \%$ to $42 \%$ of anterior infarcts but less frequently in inferior infarcts, ${ }^{11,12}$ and has been reported to occur as early as 1 day following infarction. ${ }^{11,13,14}$ The predominant features of expansion are the thinning of the ventricular wall and the regional dilatation of the infarcted area. ${ }^{11,12,15,16}$ 
Specifically, a $20 \%$ to $50 \%$ decrease in normal wall thickness has been observed with infarct expansion. ${ }^{15,17}$

The impact of reperfusion on infarct expansion has also been well studied. Hochman and $\mathrm{Choo}^{3}$ and Force et al. ${ }^{2}$ showed that reperfusion significantly reduced the incidence of infarct expansion. However, reperfusion did not have to successfully salvage myocardial tissue to inhibit expansion. Moreover, other investigators ${ }^{18}$ have also shown that reperfusion prevents ventricular dilatation and remodeling after acute myocardial infarction.

Changes in the viable, noninfarcted myocardium have also been reported following infarct expansion. Specifically, Weisman et al. ${ }^{17}$ have demonstrated that a disproportionate thinning and dilatation in the infarcted region can lead to a distortion in the shape of the entire heart. These changes, however, were evident 3 days following the infarction and can be attributed to the increased wall stress (from Laplace's law) placed on the viable myocardium because of the dilatation and thinning in the infarcted region. In a subsequent study, Weisman et al. ${ }^{19}$ demonstrated that the thinning in the noninfarcted region of the ventricle is the result of cell slippage, providing support for the hypothesis that this finding may be related to the increased wall stress.

Myocyte slippage has now become the commonly accepted mechanism responsible for infarct expansion. Erlebacher et al. ${ }^{20}$ have shown that myocardial infarction causes systolic stretching in the ischemic zone that results in stretching of the sacomeres, thinning of the myofibers, and lengthening of the ischemic segments. Subsequently, Olivetti et al. ${ }^{21}$ investigated the underlying mechanisms involved in the process of infarct expansion in a rat model. They showed that large infarcts resulted in abnormal ventricular loading that predominantly affects the diastolic portion of the cardiac cycle. This change results in an increase in diastolic stress that causes side-toside slippage of myocytes and cell lengthening.

Immediate changes in ischemic myocardium. Regional changes in acutely ischemic LV myocardium have also been well characterized in previous studies using sonomicrometry techniques. Theroux et al. ${ }^{7,8}$ previously examined end-diastolic segment lengths and showed that after 5 minutes of occlusion of the left anterior descending artery, an $11.5 \%$ increase in the end-diastolic segment length was observed in the central ischemic zone and a $4.1 \%$ increase occurred in the marginal zone end-diastolic segment length. This increase in the diastolic segment lengths was attributed to passive elongation and elastic recoil in the ischemic muscle. Furthermore, Theroux et al. ${ }^{7,8}$

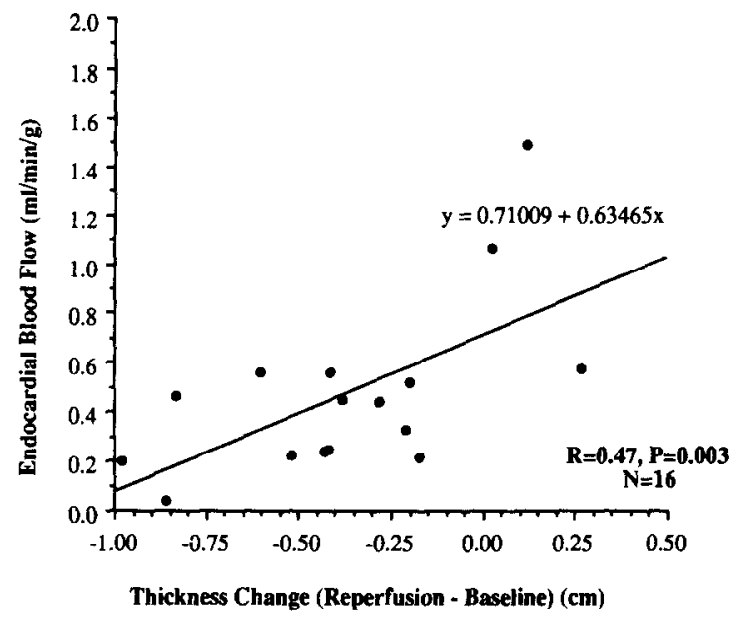

Fig. 5. Correlation of subendocardial central ischemic zone blood flow at end-reperfusion with the change in myocardial thickness from baseline to reperfusion.

showed a $2.6 \%$ increase in the normal zone end-diastolic segment length. In a clinical, echocardiographic study, Force et al. ${ }^{2}$ also observed diastolic infarct expansion several hours after coronary artery occlusion that was subsequently reversed by late reperfusion.

In the present study, both the end-diastolic endocardial and epicardial segment lengths were significantly increased following the occlusion period, which is comparable with findings in traditional infarct expansion and ischemic sonomicrometry studies. However, ischemic expansion did not produce the marked thinning of the ischemic region observed with infarct expansion. Rather, the thickness of the ischemic myocardium correlated with the residual regional myocardial blood flow from collaterals. Specifically, it was observed that when the ischemic zone blood flow was greatly reduced from baseline values, there was an accompanying thinning in the ischemic segment. This would suggest that the thickness of the ischemic segment in ischemic expansion is determined by the degree of ischemia to which the myocardium is subjected. By further comparing global and regional data, we found that ischemic expansion correlated with $\mathrm{LV}$ dilatation following the onset of ischemia. Since this correlation was not evident in the normal zone ( $r=0.1, p=\mathrm{NS})$, this would suggest that regional ischemic expansion is the major contributor to global LV dilatation evident following severe ischemia. However, there was a trend toward an increase in the endocardial segment length in the normal zone $(p=0.10)$, suggesting that ventricular remodeling also begins early in the course of an acute myocardial infarction, but progresses slower than ischemic expansion.

Reperfusion reversed the regional dilatation in the 
ischemic region, restoring the segment length to its baseline values and decreasing the $\mathrm{I} V$ dilatation. Furthermore, the reduction in regional myocardial dilatation correlated highly with the reduction in $\mathrm{LV}$ dilatation, further suggesting that the central ischemic regional dilatation was largely responsible for the $\mathrm{LV}$ dilatation. This reversal was similar between the various reperfusion durations that were used in this study ( 2,3 , and 4 hours). Therefore the reversal of ischemic expansion occurs within the first hours of reperfusion and is related to the restoration of blood flow to the previously ischemic tissue.

The present study was not specifically designed to examine the mechanisms responsible for ischemic expansion. Clearly, the severity of myocardial ischemia and the effectiveness of the subsequent reperfusion are major components of ischemic expansion and its reversal. This was evident from the fact that the thickness of the ischemic myocardium was found to closely correlate with the regional central ischemic blood flow. However, other biophysical factors may also be involved in the underlying mechanisms that result in ischemic expansion.

One possible hypothesis is that the decrease in blood flow ultimately depletes the high energy phosphate stores in the ischemic myocardium, which results in the cell's inability to continue cross-bridge cycling. The subsequent increase in end-diastolic wall stress may result in a slippage of the myofibrils which, in turn, results in sarcomere lengthening. Upon reperfusion, high energy phosphates are returned to the region and, despite reperfusion injury to the region, the cell regains its ability to actively undergo cross-bridge cycling. Furthermore, the increase in stiffness of the reperfused region as a result of the formation of edema may also protect the sarcomeres from being stretched by the stresses of the myocardium. Clearly, further studies will be necessary to explore these potential pathophysiologic mechanisms of ischemic expansion.

Advantages of quantitative two-dimensional echocardiography. This study demonstrates the use of quantitative two-dimensional echocardiography to examine segmental changes in the ischemic ventricle. Essentially, the two-dimensional echocardiographic analysis method was able to quantify changes in regional segment lengths of the left ventricle. The data obtained in this study were similar to the sonomicrometry results obtained by Theroux et al. ${ }^{7,8} \mathrm{Be}-$ cause two-dimensional echocardiography can also evaluate global function, this study was able to extend the observations of Theroux et al. by making direct comparisons between ischemic and nonis- chemic regions of the ventricle, and in turn, their relationship to global changes and function. These advantages support the use of serial two-dimensional echocardiographic examinations to assess the importance of ischemic expansion in future clinical studies.

Clinical implications. Clearly, ischemic expansion that occurs within hours of myocardial infarction needs to be distinguished from infarct expansion that is a late phenomenon and ventricular remodeling that occurs days to weeks following infarction. However, the topographic deformation produced by ischemic expansion may have significant hemodynamic consequences in the early clinical course of acute myocardial infarction. The concept of ischemic expansion may further explain the clinical findings of hemodynamic instability and heart failure that occur in a proportion of patients with acute myocardial in farction. It may be that the extent of ischemic expansion is related to the size of the risk area. Thus patients with a large ischemic bed related to the occlusion of a major coronary vessel may have greater ischemic expansion, further explaining their hemodynamic instability. Conversely, our observations of reversal of ischemic expansion with coronary reperfusion may explain the clinical observation of improved hemodynamic status and improvement in heart failure in patients following thrombolytic therapy and coronary recanalization.

Limitations. This study represents an initial effort to characterize ischemic expansion. The question of whether ischemic expansion predicts infarct expansion or ventricular remodeling has not been addressed. Further experiments involving a long-term occlusion model with serial echocardiography will be needed to answer this question. Furthermore, the infuence of changes in afterload on ischemic expansion was not assessed in these experiments. However, previous experiments in our laboratory have suggested that afterload changes do not acutely affect left ventricular flow-function relations. ${ }^{22}$

Conclusions. The present study demonstrates that the quantitative two-dimensional echocardiographic measurement of regional myocardial segment lengths is able to detect ischemic expansion. After 2 hours of coronary artery occlusion, the ischemic endocardial and epicardial end-diastolic segment lengths are significantly increased and the thickness of the ischemic segment is related to the collateral blood flow in that region. Furthermore, our data suggest that the regional dilatation in the ischemic region is the major cause for the LV dilatation that is observed following severe ischemia, and that reperfusion promptly reverses ischemic expansion and $L V$ dilatation. 


\section{REFERENCES}

1. Hutchins GM, Bulkley BG. Infarct expansion versus extension: two different complications of acute myocardial infarction. Am J Cardiol 1978;41:1127-32.

2. Force T, Kemper A, Leavitt M, Parisi AF. Acute reduction in functional infarct expansion with late coronary reperfusion: assessment with quantitative two-dimensional echocardiography. J Am Coll Cardiol 1988;11:192-200.

3. Hochman JS, Choo H. Limitation of myocardial infarct expansion by reperfusion independent of myocardial salvage. Circulation 1987;75:299-306.

4. Tennant $\mathrm{R}$, Wiggers $\mathrm{CH}$. The effect of coronary occlusion on myocardial contraction. Am J Physiol 1935;112:351-61.

5. Akaishi M, Weintraub WS, Schneider RM, Klein LW, Agarwal JB, Helfant RH. Analysis of systolic bulging: mechanical characteristics of acutely ischemic myocardium in the conscious dog. Circ Res 1986;58:209-17.

6. Noma S, Askenase AD, Agarwal JB, Helfant RH. The effect of changes in afterload on systolic bulging. Circulation 1988; 77:221-6.

7. Theroux P, Ross J Jr, Franklin D, Kemper WS, Sasayama S. Regional myocardial function in the conscious dog during acute coronary occlusion and response to morphine, propranolol, nitroglycerine, and lidocaine. Circulation 1976;53:30214.

8. Theroux P, Franklin D, Ross J Jr, Kemper WS. Regional myocardial function during acute coronary artery occlusion and its modification by pharmacologic agents in the dog. Circ Res 1974;35:896-908.

9. Buda AJ, Zotz RJ. Serial assessment of circumferential regional left ventricular function following complete coronary occlusion. Am HearT J 1986;112:447-52.

10. Buda AJ, Zotz RJ, Pace DP, Krause LC, Turla M. Immediate rebound followed by deterioration of regional left ventricular function with coronary reperfusion. J Am Coll Cardiol 1986; 8:333-41.

11. Meizlisch JL, Berger HG, Plankey M, et al. Functional left ventricular aneurysm formation after acute anterior transmural infarction: incidence, natural history, and prognostic implications. N Engl J Med 1984;311:1001-6.
12. Eaton LW, Weiss JL, Weisfeldt ML, Bulkley BH. Early dilatation of the infarcted segment in acute transmural myocardial infarction: role of infarct expansion in acute left ventricular enlargement. J Am Coll Cardiol 1984;4:201-8.

13. Eaton LW, Bulkley BH. Expansion of acute myocardial infarction: its relationship to infarct morphology in a canine model. Circ Res 1981;49:80-8.

14. Hochman JS, Bulkley BH. Expansion of acute myocardial infarction: an experimental study. Circulation 1982;65:1446-50.

15. Hutchins GM, Bulkley BH. Infarct expansion versus extension: two different complications of acute myocardial infarction. Am J Cardiol 1978;41:1127-32.

16. Jugdutt BI, Michorowski BL. Role of infarction expansion in rupture of the ventricular septum after acute myocardial infarction: a two-dimensional echocardiographic study. Clin Cardiol 1987;10:641-52.

17. Weisman HF, Bush DE, Mannisi JA, Bulkley BH. Global cardiac remodelling after acute myocardial infarction: a study in the rat model. J Am Coll Cardiol 1985;5:1355-62.

18. Lavie CJ, O'Keefe JH, Cheseboro JH, Clements IP, Gibbons RJ. Prevention of late ventricular dilatation after acute myocardial infarction hy successful thrombolytic reperfusion. Am J Cardiol 1990;66:31-6.

19. Weisman HF, Bush DE, Mannisi JA, Weisfeldt ML, Healy B. Cellular mechanisms of myocardial infarct cxpansion. Circulation 1988;78:186-201.

20. Erlebacher JA, Fichter RC, Alonso DR, Devereux RB, Gay WA Jr. Early infarct expansion: structural or functional? J Am Coll Cardiol 1985;6:839-44.

21. Olivetti G, Capasso JM, Sonneblick EH, Anversa P. Side-toside slippage of myocytes participates in ventricular wall remodeling acutely following myocardial infarction in rats. Circ Res 1990;67:23-34.

22. Kavanaugh KM, Brenner HM, Gallagher KP, Buda AJ. Effect of afterload alterations on the functional border zone measured with two-dimensional echocardiography during acute coronary occlusion. AM HEART J 1988;116:942-53. 